\title{
Relationship between risk factors and coronary heart disease in premenopausal and postmenopausal women
}

\author{
Grupo de Coronária do Instituto do Coração do Hospital \\ das Clínicas - FMUSP
}

Coronary heart disease is the main cause of death among women in developed regions in Brazil with a enormous public health costs. The incidence of coronary heart disease increases after the menopause, resulted from a marked ovarian deficiency in producing endogenous estrogen. It is also well estabilished the low rate of coronary artery disease in premenopausal and in postmenopausal women receiving hormone replacement therapy. On the other hand, the prognosis after myocardial infarction, coronary angioplasty, and coronary bypass surgery is worse in women than among men. It is not well known the cause of this excess mortality. This fact may be related to the sex differences in access to high-quality medical therapy and strategies to women. Thus, best approaches are needed, especially in postmenopausal women who have an increased risk for cardiovascular diseases.

The primary prevention of coronary heart disease in women envolves changes of these most important modifiable risk factors: smoking, hypercholesteromia, diabetes melito and hypertension. Smoking and diabetes melito are the most important risk factors in premenopausal women. Previous studies have shown greater prevalence of cardiovascular diseases among who smoke, a straight relation with the number of cigarrete per day and the association with other risk factors. Smoking increased among young and disadvantaged women, and was associated with an early natural menopause. High prevalence of cardiovascular diseases was also observed in young women using contraceptives drugs, speccialy in smoking women. The contraceptives and cigarrete smoking, synergistycally, are associated with adverse effects in lipid profile, coagulation system, and platelet aggregation. Although, consistent evidences werenot supported with contraceptives with low doeses of strogen. In fact, strogen in no smoking women promotes a natural protection against cardiovascular diseases. The thrombus formatiom and atheromatous plaque development are favorated by smoking-induced extensive endothelial damage that enhance platelet activation with release of grouth factors, raises serum level of fibrinogen, and decreases high-density lipoprotein. Though, the pathophysiology of coronary heart disease in premenopausal smoking women those who use contraceptives pro- bably accounts more for a thrombogenic than a atheogenic process. Furthermore, smoking has a anti-estrogenic effect, increasing the hydroxilation of carbon-2 from estradiol, and reducing the carbon- 16 hydroxilation. The former pathway generates metabolites with less potent estrogen effects.

The risk of cardiovascular diseases is also increased in young diabetic women with a direct relationship between chronic hypoglicemia detected by sustained high plasma levels of glycosilated hemoglobin. In our experience, the prevalence of diabetes melito insulin-dependent was greater in premenopausal women. Because diabetes damages the endothelium, the natural protection of intact endothelium is compromissed, by reduced autocrine vasodilators production, or even those related to the impaired effect of estrogen on injured vascular tissue. It was also observed the frequent association of others risk factors among diabetic women. Although, a minority of premenopausal women are afflicted with premature atherosclerosis, smoking cessation and better control of hipertension and plasma glycemia levels may have an important impact on the incidence of cardiovascular diseases in young females.

The prevalence of smokinfg and diabetes melito are lower in postmenopausal than in premenopausal women. However, there are an abrupt increase in the incidence if coronary heart disease in postmenopausal women, probably by losing the atheroprotection confered by the estrogens, associated by the increased prevalence of hipertension and dyslipitemia. Epidemiologic evidence indicates almost 50\% in the incidence of cardiovascular disease in postmenopausal women receiving estrogen therapy. The hormonal replacement seems to improve the cholesterol profile, the low density lipoprotein anti-oxidation, the endothelium-derived vasoactive substances, and the performance on exercise-induced miocardial ischemia. Therefore, besides risk factors control by changing some lifestiles, as the diet, smoking, and physical inactivity, and treating the diseases associated with coronary heart disease, it is wise to introduce estrogen replacement therapy in postmenopausal women, in order to enhance both the quality and the life expectancy. 